\title{
Extended Patient Survival after Inferior Vena Cava Stent Placement for Obstruction Caused by Metastatic Colon
}

\section{Cancer}

\author{
Huk $\mathbf{M}^{1}$, Emmerton Coughlin $\mathrm{HM}^{2}$ and Saltman $\mathrm{DL}^{3 *}$ \\ ${ }^{1}$ Department of Diagnostic Imaging, Royal Jubilee Hospital, Victoria, Canada \\ ${ }^{2}$ Department of Surgery, Royal Jubilee Hospital, Victoria, Canada \\ ${ }^{3} \mathrm{BC}$ Cancer Agency, Victoria, Canada
}

\section{Case Report}

Volume 3 Issue 4

Received Date: September 13, 2019

Published Date: September 19, 2019

DOI: $10.23880 /$ crij-16000155

*Corresponding author: David L Saltman, MD, PhD, BC Cancer Agency, 2410 Lee Avenue, Victoria, British Columbia, V8R 6V5, Canada, Tel: 250-519-5575; Fax: 250-519-5595; Email: david.saltman@bccancer.bc.ca

\begin{abstract}
Venous obstruction by malignancy is a significant cause of morbidity. Stenting of superior or inferior vena cava obstruction can be very effective in improving symptoms. In some cases, patients who are considered palliative may become eligible for active cancer treatment as a result of the relief of their venous obstruction. In this report, we describe the case of a patient with advanced colon cancer with a large right hepatic lobe metastatic lesion severely compressing the suprarenal inferior vena cava (IVC) resulting in extensive venous collaterals, abdominal wall distension and edema of both lower extremities. Initial treatment with chemotherapy were unsuccessful. The patient was deemed not suitable for further treatment due to her poor performance status. A $24 \mathrm{~mm}$ x $70 \mathrm{~mm}$ Wallstent venous endoprosthesis was inserted under ultrasound guidance resulting in a rapid improvement in the patient's symptoms of abdominal pain and anasarca. The patient was restarted on chemotherapy one month after the stent insertion resulting in a significant decrease in the size of the dominant right hepatic lobe metastasis. The stent remains patent 7 months after insertion. The patient continues with active treatment of her cancer and maintains an excellent quality-of-life.
\end{abstract}

Keywords: Vena Cava; Stent; Hepatic Metastases; Colon Cancer

Abbreviations: IVC: Inferior Vena Cava; SVC: Superior Vena Cava; CT: Computerized Tomography.

\section{Introduction}

Compression of the inferior vena cava (IVC) by malignancy has been reported for a variety of histologies and is most commonly seen in advanced metastatic disease [1-3]. The symptoms and signs of inferior vena cava syndrome will depend on the severity of the vena cava compression and resulting pressure gradient. Severe occlusion results in ascites and anasarca below the diaphragm. Imaging with computerized tomography (CT) scans and venography will demonstrate the IVC 


\section{Clinical Radiology \& Imaging Journal}

compression and often show collateral venous distention. Unlike the more commonly seen superior vena cava (SVC) obstruction where chemotherapy and radiation-sensitive primary lung tumors are frequently involved, tumors that cause IVC obstruction may be less sensitive to radiation or the location of the tumor may make radiation therapy unsafe. Chemotherapy may also be unsafe in this setting where hepatic and renal function can be compromised, and the patients often have a poor performance status. Expandable, intraluminal stent placement is an option for the treatment for malignant IVC compression; however, they are usually deployed in a palliative setting with very low median overall survivals after the stents are deployed [2-7]. In this report, we describe a case of extended patient survival and patency of an IVC stent after placement of the endoprosthesis for severe compression of the IVC from a large right hepatic lobe colon cancer metastasis.

\section{Case History and Methods}

A 54 -year-old female presented with abdominal discomfort, a change in her bowel habits and hematochezia. She had a history of hereditary spherocyto sis and required a splenectomy at age thirteen. A CT scan demonstrated multiple masses in both lobes of the liver with the largest being $16 \mathrm{~cm}$ in the right lobe. A circumferential non-obstructing mass was noted in the sigmoid colon. A flexible sigmoidoscopy confirmed the present of mass in the sigmoid colon. Biopsies revealed an adenocarcinoma consistent with a colon primary. The physical examination revealed distended and painful abdomen but no edema.

The patient was started on single-agent oral capecitabine chemotherapy but five weeks later had increasing abdominal pain. A repeat CT scan showed progression of the liver metastases with the dominant right lobe mass now measuring $18 \mathrm{~cm}$ in greatest diameter and severely compressing the suprarenal, intrahepatic IVC (Figure 1). A trace of intraabdominal free air was noted suggesting a microperforation of the colon. The patient was taken to surgery and underwent an open sigmoid resection and Hartmann's procedure. Postoperatively, the patient's abdominal wall pain and edema of the lower extremities increased significantly. The serum creatinine and total bilirubin remained in the normal range.
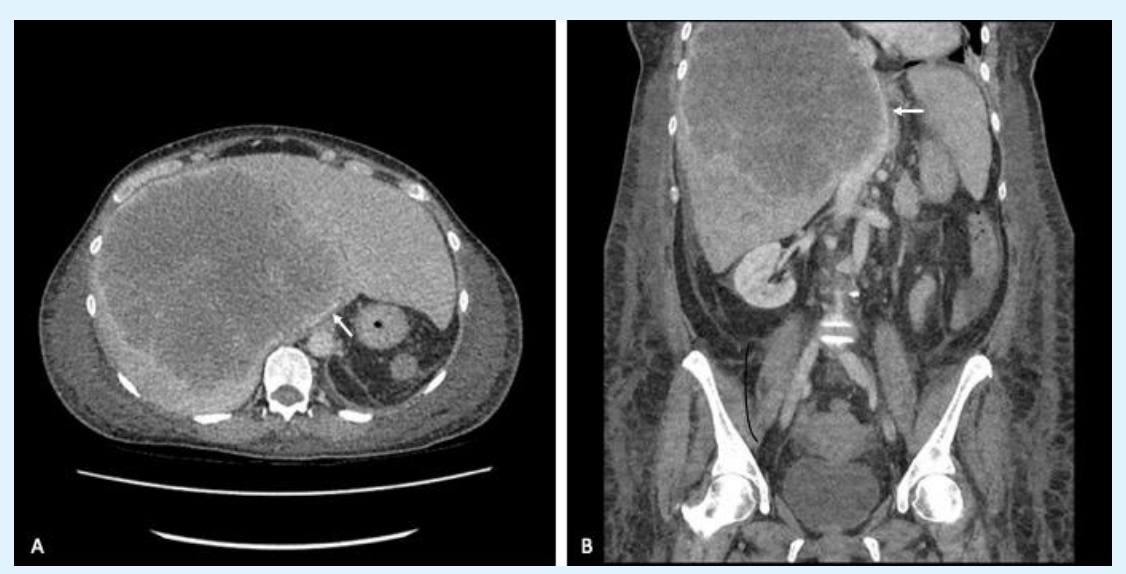

Figure 1: Axial (A) and coronal (B) CT scan images of the abdomen ten days prior to IVC stenting demonstrating the severe compression of the IVC (arrow).

After discussion with the patient and her family, a decision was made to insert an IVC stent to palliate her symptoms. The right common femoral vein was accessed with a 16-French $30 \mathrm{~cm}$ sheath inserted utilizing ultrasound guidance. The right internal jugular vein was accessed with a 7-French sheath. Multiple venocavagrams confirmed the severe IVC narrowing and extensive venous collaterals (Figure 2A). A $24 \mathrm{~mm} \times 70 \mathrm{~mm}$
Wallstent (Boston Scientific Corporation, Natick, MA, USA) was deployed. The stent was angioplastied with a 12 mm Mustang balloon (Boston Scientific), followed by a 20 mm Atlas balloon (Bard, Tempe, AZ, USA), (Figures 2B \& C). Venocavagram after the stent placement showed a widely patent IVC and the collateral venous network was no longer visible (Figure 2D). The procedure was tolerated well without any immediate complications. 


\section{Clinical Radiology \& Imaging Journal}

Within days of the procedure the anasarca and pain improved significantly. The weight decreased by 10 kilograms in 30 days. The patient was subsequently restarted on chemotherapy using 5-fluorouracil, irinotecan and panitumumab. A follow-up CT scan five months after the insertion of the IVC stent demonstrated a decrease in the size of the dominant right hepatic lobe mass, with decreased compression of the IVC and patent stent (Figure 3). The patient remains on chemotherapy 7 months after stent insertion. The stent remains patent and the patient continues to have an excellent quality-oflife.

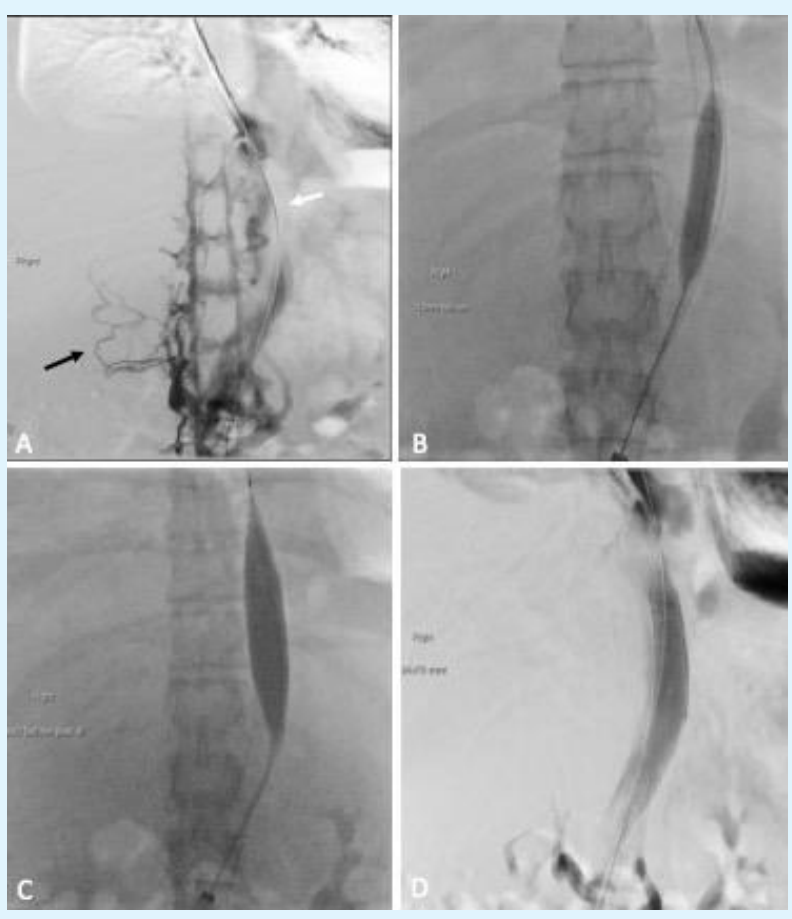

Figure 2: A: Venocavagram prior to the placement of the stent showing the severe IVC obstruction (white arrow) and collateral network of vessels (black arrow), B \& C: Angioplasty with a $12 \mathrm{~mm}$ balloon followed by a $20 \mathrm{~mm}$ balloon, D: Final venocavagram after stent placement and angioplasties demonstrating the widely patent IVC and absence of collateral veins.
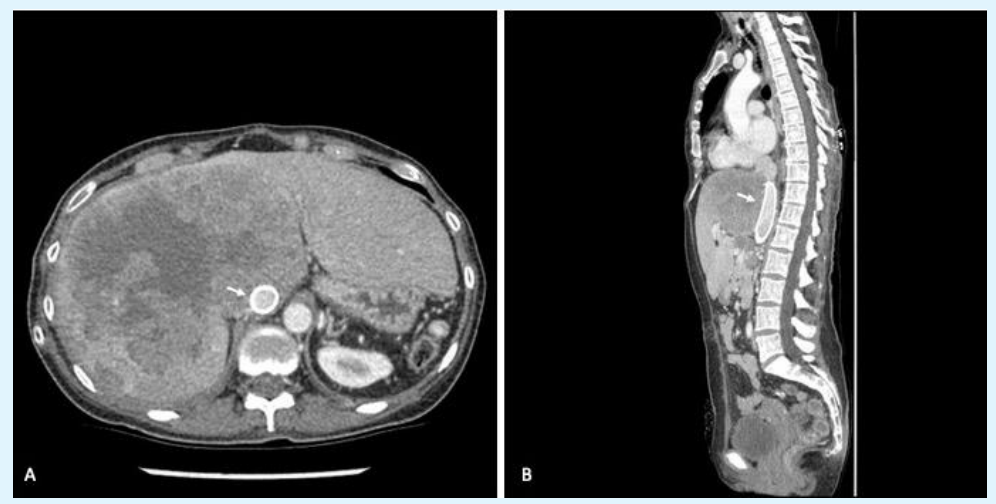

Figure 3: CT scan axial (A) and sagittal (B) images of the abdomen 5 months after IVC stent placement showing the stent (arrows) remains widely patent. 


\section{Clinical Radiology \& Imaging Journal}

\section{Discussion}

The use of expandable intraluminal grafts or stents was first reported over 30 years ago [8]. Since then, these devices have been deployed to relieve both malignant and non-malignant vena cava outflow obstruction. The most frequently used stents in the setting of malignant vena cava obstruction are the Wallstent (Boston Scientific) and the Gianturco-Z stent (Cook Medical, Bloomington, IN, USA). One criticism of the Gianturco -Z stent in malignant IVC obstruction is the low radial strength and large interstices resulting in a higher rate of reintervention because of recurrent obstruction [2]. Although the Wallstent was less likely to require reintervention in the retrospective study reported by Devcic, there was increased risk of misplacement towards the right atrium in suprarenal placements [2]. A Wallstent $20 \times 70 \mathrm{~mm}$ stent was placed in the suprarenal IVC in our patient with no procedure issues, evidence of migration towards the right atrium or other late complications 7 after insertion. Thrombosis and embolization have been reported by several authors as complications of IVC stenting [2,3,5]. Our patient was anticoagulated prior to the stent insertion and remained on prophylactic doses of low molecular weight heparin for three months after the procedure because of her high risk for venous thromboemboli due to the advanced stage of the cancer and her prior splenectomy. The frequency of other complications of IVC stent placement is difficult to determine given small numbers of reports in the literature, most of which are single case studies. In the case of the more commonly reported SVC stenting, bleeding, especially if the patients are anticoagulated, pain, sepsis, and rarely vena cava perforation, have all been reported $[9,10]$.

Unlike the obstruction of the SVC where primary small cell and nonsmall cell lung cancers are most often implicated, the obstruction of the IVC may be caused by a plethora of different histologies, with primary hepatocellular and metastatic colon cancer being the most frequently reported $[2-7,11,12]$. Patients with malignant IVC obstruction most often have advanced metastatic disease and are deemed palliative because of their poor performance status or the lack of effective therapies. Metastatic colorectal cancer even in the advanced stages is often sensitive the chemotherapy. Improvement in therapeutic regimens for advanced colorectal cancer over the last two decades has resulted in a significant improvement in survival. Most of the reported cases of malignant obstruction of the IVC due to colon cancer died shortly after stenting due to advanced disease, and other co-morbidities contributing to a poor performance status $[2,7]$. However, in the case of one patient with obstruction due to IVC thrombosis, stenting was followed by chemotherapy and a prolonged survival [13].The patient described in this report was offered an IVC stent placement as a palliative end -of-life procedure to relieve her symptoms of abdominal pain and anasarca. However, as a result of the dramatic improvement in her condition following the procedure, she was able to be discharged from hospital and resume chemotherapy.

\section{Conclusion}

We present a case of severe IVC obstruction due to compression from a large right hepatic lobe colon cancer metastasis. The clinical status improved dramatically after an endoprosthesis placement, allowing continuation of chemotherapy. Patients with colorectal cancer demonstrating signs and symptoms of IVC syndrome should be investigated and considered for IVC stenting to palliate symptoms, and in selected cases, for maximization of treatment options to improve overall survival.

\section{References}

1. Friedman T, Quencer, KB, Kishore SA, Winokur RS, Madoff DC (2017) Malignant venous obstruction: superior vena cava syndrome and beyond. Semin Intervent Radiol 34(4): 398-408.

2. Devcic Z, Techasith T, Banerjee A, Rosenberg J, Sze DY (2016) Technical and anatomic factors influencing the success of inferior vena caval stent placement for malignant obstruction. J Vasc Interv Radiol 27(9): 1350-1360.

3. Fletcher WS, Larkin PC, Pommier RC, Wilmarth $\mathrm{T}$ (1998) Results of treatment of inferior vena cava syndrome with expandible metallic stents. Arch Surg 133(9): 935-939.

4. Brountzos EN, Binkert CA, Panagiotou IE, Petersen BD, Timmermans H, et al. (2004) Clinical outcome after intrahepatic venous stent placement for malignant inferior vena cava syndrome. Cardiovasc Intervent Radiol 27(2): 129-136.

5. McGee H, Maudgil D, Tookman A, Kurowska A, Watkinson AF (2004) A case series of inferior vena cava stenting for lower limb oedema in palliative care. J Palliat Med 18(6): 573-576. 


\section{Clinical Radiology \& Imaging Journal}

6. Zamora CA, Sugiomoto $\mathrm{K}$, Mori $\mathrm{T}$, Taniguchi $\mathrm{T}$, Tsurusaki M, et al. (2005) Use of Wallstents for symptomatic relief of malignant inferior vena cava obstruction. Radiat Med 23(5): 380-385.

7. Kudo H, Yata Y, Orchara T, Kageyama M, Takahara T, et al (2006) Malignant inferior vena cava syndrome. Intern Med 45(4): 219-220.

8. Palmaz JC, Sibbitt RR, Reuter SR, Tio FO, Rice WJ (1985) Expandable intraluminal graft: A preliminary study. Work in progress. Radiology 156(1): 72-77.

9. Nguyen NP, Borok TL, Welsh J, Vinh-Hung V (2009) Safety and effectiveness of vascular endoprosthesis for malignant superior vena cava syndrome. Thorax 64(2): 174-178.

10. Da Ines $D$, Chabrot $P$, Motreff $P$, Alfidja A, Cassagnes $L$, et al. (2010) Cardiac tamponade after malignant superior vena cava stenting: Two case reports and brief review of the literature. Acta Radiol 51(3): 256259.

11. Akerman S, Kambisch A, Gucalp R, Scher L, Haigentz $M$ (2008) Inferior vena cava syndrome from pancreatic adenocarcinoma: successful symptom palliation with endovascular stenting. J Palliat Med 11(8): 1066-1068.

12. Gasparis AP, Kokkosis A, Labropoulos N, Tassiopoulos AK, Ricotta JJ (2009) Venous outflow obstruction with retroperitoneal Kaposi's sarcoma and treatment with inferior vena cava stenting. Vasc Endovascular Surg 43(3): 295-300.

13. Sato Y, Inaba Y, Yamaura H, Takaki H, Arai Y (2012) Malignant inferior vena cava syndrome and congestive hepatic failure treated by venous stent placement. J Vasc Interv Radiol 23(10): 1377-1380. 